\title{
Simulation of horizontal tau-neutrino induced shower to optimize the site parameters
}

\author{
A .YILMAZ* \\ Giresun University, Giresun 28200, TR \\ E-mail: aliyilmaz@giresun.edu.tr \\ M. IORI \\ La Sapienza, Univeristy of Rome, Piazzale A. Moro 5, Rome 00185, IT \\ E-mail: maurizio.iorieromal.infn.it \\ H. DENIZLI ${ }^{\dagger}$ \\ Abant Izzet Baysal University, Bolu 14280, TR \\ E-mail: denizli_h@ibu.edu.tr
}

\section{K. YUKSEL OYULMAZ}

Abant Izzet Baysal University, Bolu 14280, TR

E-mail: kaan. oyulmaz@gmail.com

\section{S. ATIK YILMAZ}

Abant Izzet Baysal University, Bolu 14280, TR

E-mail: seyma.atik@ogr.ibu.edu.tr

\section{U. KESKIN}

Abant Izzet Baysal University, Bolu 14280, TR

E-mail: umut.kesinecern.ch

\section{J. RUSS}

Carnegie-Mellon University, Pittsburgh, PA 15213, USA

E-mail: russaheps.phys. cmu.edu

\begin{abstract}
In the neutrino physics the direct measurement of the cosmogenic neutrino at energy of $10^{15}-$ $10^{18} \mathrm{eV}$ is still a challenging item. The most recent analysis of IceCube data results in an energy spectrum between $10^{13}$ and $10^{16} \mathrm{eV}$. We present the preliminary results of detailed Monte Carlo simulation of tau-induced air shower. In this study we consider the highest tau decay branching ratio $\left(\pi^{-} \pi^{0}\right)$ at different energies $\left(10^{16}-10^{18} \mathrm{eV}\right)$ generated by CORSIKA to evaluate the detection efficiency of the TAU-shoWER (TAUWER) array. It is designed as a future experiment for Ultra High Energetic tau-neutrino (UHE $v_{\tau}$ ) astronomy to use Earth neutrino skimming strategy. The parameters (the distance from array plane to the emerging point and the neutrino energy) to optimize the detection efficiency and the trigger design for the TAUWER array setup are presented.
\end{abstract}

35th International Cosmic Ray Conference - ICRC2017

10-20 July, 2017

Bexco, Busan, Korea
* Speaker.
${ }^{\dagger}$ Corresponding author 


\section{Introduction}

In order to get a detectable rate of neutrino flux, very large scale (surface or volumetric) detector array is needed since the neutrino flux is low and the detection probability is so small. For this purpose, there are many experiments constructed such as MAGIC[1], VERITAS[2], H.E.S.S[3], KASCADE-Grande[4], IceCube[5], CTA[6], LOPES[7], LOFAR[8], with using various detection techniques (Cherenkov, air fluorescence, radio waves). Recently, Icecube collaboration reported 28 neutrino candidate events in the energy range of 30 to $1200 \mathrm{TeV}$ [9]. TAUWER array experiment, aims to detect UHECRs and identify UHE $(\sim 0.1-100 \mathrm{EeV})$ tau-neutrino $\left(v_{\tau}\right)$, is capable of measuring large zenith angle cosmic rays as well as detecting the signature of UHE $v_{\tau}$ interactions using the "Earth Skimming" strategy. In this strategy, $v_{\tau}$ are expected to be observed trough the detection of showers induced by the decay of emerging $\tau$ leptons generated by $v_{\tau}$ interaction in the Earth $[10,11]$. In order to increase the detection probability of Earth skimming $v_{\tau}$ shower, TAUWER array is aligned to the direction of the shower on the surface of the mountain (see section 2).

The evaluation of air showers can identify the primary cosmic rays by distinguishing its energy and the arrival directions. A ground based scintillation array can generally give only the statistical information about the secondary particles of the shower. Density of the detected particles on the array is correlated with the energy of primary cosmic ray and the development of the shower from first injection point to detected array plane.

In this study, we present the preliminary results of Monte Carlo (MC) simulation of event rate (/ density) triggered by Earth skimming $v_{\tau}$ carried out for a possible TAUWER array configuration layout in case of the candidate site. In addition, we study the simulation of trigger and identification probability of the showers on the array layout.

The outline of this paper, the array configuration layout used in the data production described in section 2. In section 3, Monte Carlo simulation study is given. Trigger and identification studies for $\tau$-induced shower as a function of $\tau$ lepton energy and shower development length are given in section 4 .

\section{Description of the array}

The module was designed to recognise single particles and determine the direction of motion (up/down) and measure the trajectory angles. The hardware of the detector prototype consists of two identical scintillator counters, named tower, separated by $160 \mathrm{~cm}$ apart [12]. Each counter consisting of an organic scintillator panel $20 \times 40 \mathrm{~cm}^{2}, 1.4 \mathrm{~cm}$ thick as seen in Fig. 1 at the upper left corner. The signal in the scintillator panel read by one SensL silicon photomultipler $\left(3 \times 3 \mathrm{~mm}^{2}\right)$. This SiPM has short output pulse of $<2$ ns at FWHM, and the gain is $\sim 10^{6}$ which permits us to use the time of flight (TOF) method [13]. The aim of this detector is select horizontal tracks to detect tau shower produced by the neutrino interacting in Earth crust. So this can be achieved with TOF resolution of $0.5 \mathrm{~ns}$ without using any shielding material.

The array consists of 640 stations placed on the $30^{\circ}$ inclined plane (mountain surface) in the matrix form of 16 rows ( $\mathrm{Z}^{\prime}$-axis) and 40 columns (Y-axis) which are separated by $30 \mathrm{~m}$ apart. $\mathrm{YZ}^{\prime}$ - 
plane is the projection of $\mathrm{YZ}$ plane on the inclined surface. This setup covers approximately 0.5 $\mathrm{km}^{2}$ on the mountain surface, see Fig. 1.

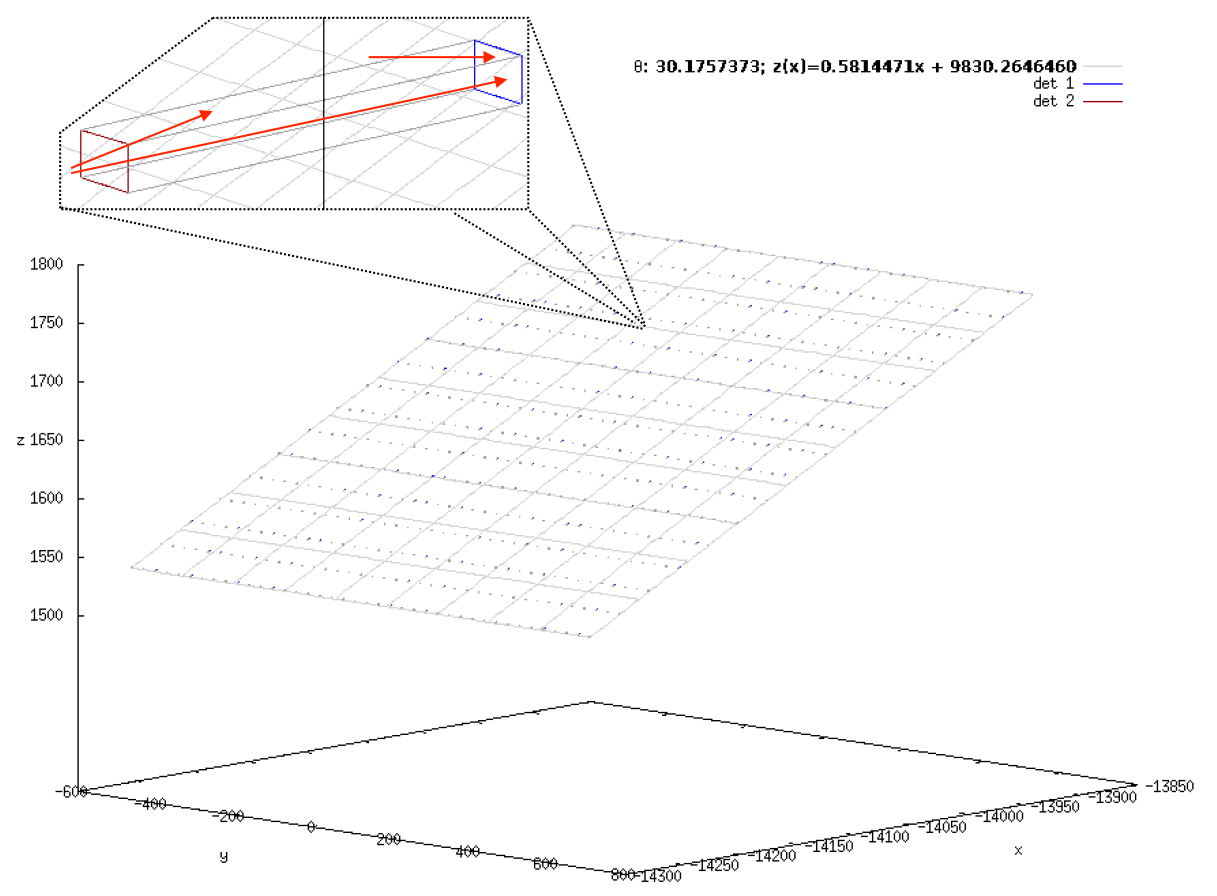

Figure 1: The sketch of TAUWER array with 640 stations (shown as dot) on the $30^{\circ}$ inclined plane (drawn as grid). The blow-up image shows one station consisting of 2 detectors separated by $160 \mathrm{~cm}$ apart looking $\sim 2^{o}$ downward from the horizontal axis. The red arrow represents the tracks counted as "hit" on each detector.

\section{Monte Carlo Simulations}

Since the goal of this study is to detect UHE $v_{\tau}$, the TAUOLA program[15] is used to generate all possible decay mode with the energy range of $10^{15}-10^{18} \mathrm{eV}$. The decay modes of $\tau$ lepton with their probabilities and their secondaries are given in Table $1 . \pi^{-} \pi^{0}$ with a $25.8 \%$ branching ratio is chosen to study the optimization and identification performance of the expected $v_{\tau}$ induced showers by TAUWER array. CORSIKA[17] is used to simulate showers with 4 development lengths $(3 \mathrm{~km}$, $5 \mathrm{~km}, 7 \mathrm{~km}$ and $10 \mathrm{~km}$ ), grouped in 5 different energies (about $10^{7} \mathrm{GeV}, 5 \times 10^{7} \mathrm{GeV}, 10^{8} \mathrm{GeV}$, $5 \times 10^{8} \mathrm{GeV}$ and $10^{9} \mathrm{GeV}$ ) and 9 showers in each combinations.

CORSIKA (version 6.99) was modified and compiled to simulate very inclined $\left(>90^{\circ}\right)$ shower and observe it at an inclined plane ( $30^{\circ}$ from the ground). The simulations were started with selecting QGSJETII[18] and GEISHA for high and low energy interaction models, respectively. In order to make more realistic simulation for correctly include the atmospheric depth, "CURVED EARTH" and "SLANT" options were activated. To read and convert CORSIKA binary files "COAST" option was also selected. Injection point of the produced air showers, induced by $\tau$ lepton, was set to 1500 $\mathrm{m}$ a.s.l. and developed up to the detector level (2250 $\mathrm{m}$ a.s.1.). In order to minimize the showerto-shower variations nine showers were generated for each energy, each development length and 
Table 1: $\tau$ decay channels with its probabilities, taken from[16].

\begin{tabular}{llll}
\hline \hline Decay & Secondaries & Branching Ratios & Air Shower \\
\hline$\tau \rightarrow \mu^{-} \bar{v}_{\mu} v_{\tau}$ & $\mu^{-}$ & $\sim 17.4 \%$ & Unobservable \\
$\tau \rightarrow e^{-} \bar{v}_{e} v_{\tau}$ & $e^{-}$ & $\sim 17.8 \%$ & 1 Electromagnetic \\
$\tau \rightarrow \pi^{-} v_{\tau}$ & $\pi^{-}$ & $\sim 11.8 \%$ & 1 Hadronic \\
$\tau \rightarrow \pi^{-} \pi^{0} v_{\tau}$ & $\pi^{-}, \pi^{0} \rightarrow 2 \gamma$ & $\sim 25.8 \%$ & 1 Had., 2 Elect. \\
$\tau \rightarrow \pi^{-} 2 \pi^{0} v_{\tau}$ & $\pi^{-}, 2 \pi^{0} \rightarrow 4 \gamma$ & $\sim 10.79 \%$ & 1 Had., 4 Elect. \\
$\tau \rightarrow \pi^{-} 3 \pi^{0} v_{\tau}$ & $\pi^{-}, 3 \pi^{0} \rightarrow 6 \gamma$ & $\sim 1.23 \%$ & 1 Had., 6 Elect. \\
$\tau \rightarrow \pi^{-} \pi^{-} \pi^{+} v_{\tau}$ & $2 \pi^{-}, \pi^{+}$ & $\sim 10 \%$ & 3 Had. \\
$\tau \rightarrow \pi^{-} \pi^{+} \pi^{-} \pi^{0}$ & $2 \pi^{-}, \pi^{+}, \pi^{0} \rightarrow 2 \gamma$ & $\sim 5.18 \%$ & 3 Had., 2 Elect. \\
\hline
\end{tabular}

for the highest probable decay mode. The computing time of showers was increased in accordance with increasing energy (from $10^{7} \mathrm{GeV}$ to $10^{9} \mathrm{GeV}$ ) since the "thinning" strategy was not used in shower productions. At the end of simulations, an offline program written (in $C++$ ) to study the array performance. CORSIKA output file includes not only position ( $\mathrm{x}, \mathrm{y}$ and $\mathrm{z}$ ) information but also momentum (Px, Py, Pz, and Ptot) information of each particle on inclined place. Using this informations the trajectory is reconstructed for each particle and counted when it passes trough the tiles.

Average number of total $e^{ \pm}$detected in the array for a shower as a function of development length for 5 different energies is given Fig. 2(a), while average number of $e^{ \pm}$in the central station is in Fig. 2(b). In both figure, it is clearly seen that shower reaches the maximum size between 5 $\mathrm{km}$ and $7 \mathrm{~km}$ development lengths. At $3 \mathrm{~km}$ in all energies, the density is lower than 5-7 km development lengths which indicates the shower is still developing. The density at $10 \mathrm{~km}$ development length is also lower than 5-7 km since particles in the shower has been annihilated before reaching to the stations. Since the detected particle density is higher at $5-7 \mathrm{~km}, 7 \mathrm{~km}$ development length is selected and concentrated on subarray $(4 \times 4)$ around the shower core. As seen from this figure, the showers can be identified easily in terms of energy and shower development length.

Shower width in both $\mathrm{Y}$ and $\mathrm{Z}^{\prime}$ axis on the observation plane is defined by the distance between the farthermost stations in which at least 5 hits is required. Fig. 3 shows the average width of the showers for $\pi^{-} \pi^{0}$ decay mode at $7 \mathrm{~km}$ development length as a function of energy. Each point is the average of 9 showers generated approximately the same energy. To find a correlation between the shower width and its energy, the power law function was applied. As seen from the fit result, shower width in $\mathrm{Y}$-axis is given by $0.0565 \times E^{0.3936}$ while in $\mathrm{Z}^{\prime}$ axis $0.12 \times E^{0.3607}$, where $\mathrm{E}$ is the primary particle energy.

\section{Trigger Decision}

To study the trigger efficiency of $\tau$-induced shower on the TAUWER array, Trigger Probability, $\operatorname{TP}\left(\theta, E_{i}, \Delta X, D_{M}\right)$ function is defined as the number of fired stations over all the stations in the array for zenith angle differs from $92.0^{\circ}$ to $92.5^{\circ}$, with initial primary energy $E_{i}(\mathrm{GeV})$, shower development length $\Delta X(\mathrm{~km})$ is from first originated point to the array position and decay mode 


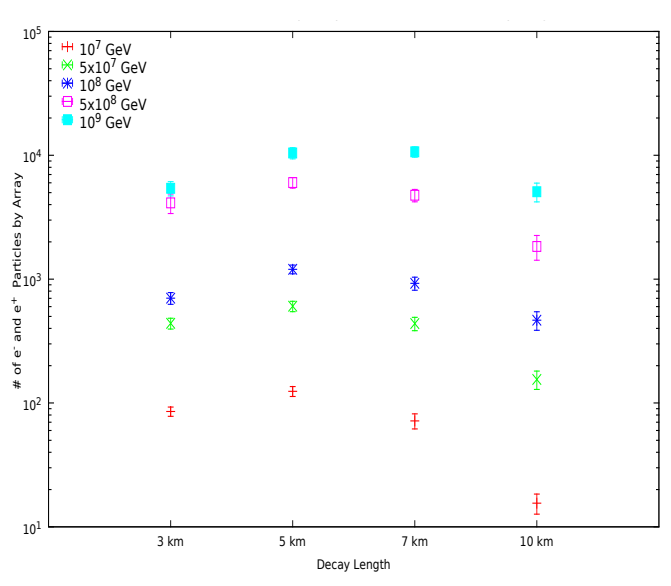

(a)

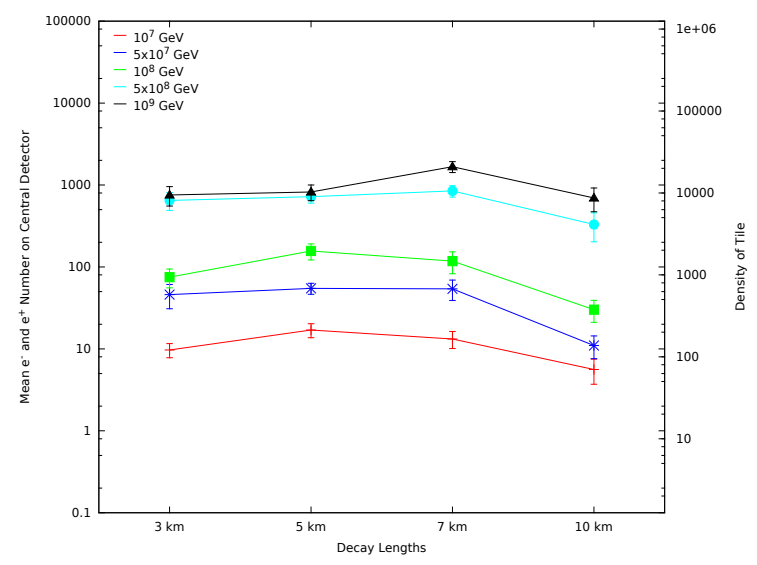

(b)

Figure 2: Average number of total $e^{ \pm}$detected on the array as a function of shower development length for different energies $\left(10^{7}, 5 \times 10^{7}, 10^{8}, 5 \times 10^{8}, 10^{9}\right)$ at $\pi^{-} \pi^{0}$ decay mode. (a) for the array $(16 \times 40$ stations $)$, (b) for the central station.
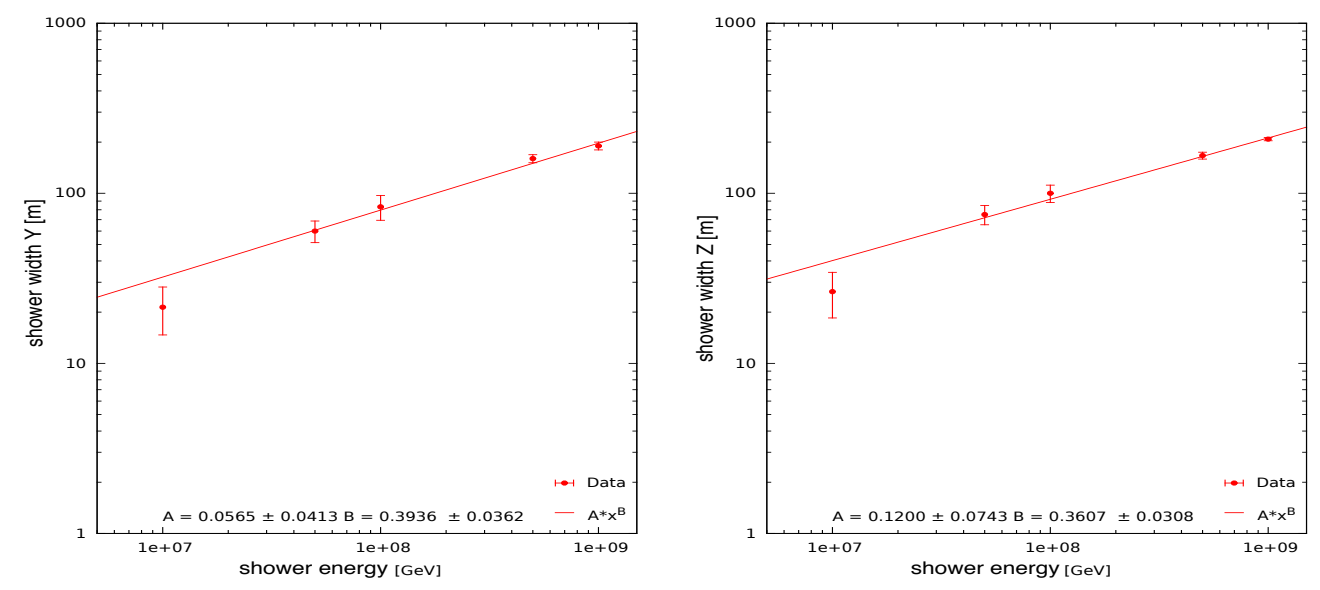

Figure 3: Shower widths on the inclined plane ( $\mathrm{Y}$ on the left side, $\mathrm{Z}$ on the right side) as a function of shower energy on the array at fixed shower development length $(7 \mathrm{~km})$ for $\pi^{-} \pi^{0}$ decay mode.

used in the analysis, $\mathrm{D}_{M}$. The general trigger $(\boldsymbol{T 1})$, is given by the hits must be inside the main time window $(4.26 \mu s)$ defined by array geometry. Due to the fact that we have a background to select $\tau$-induced shower we define a trigger, close number of stations with many hits per station in a given time window. To define these parameters (number of stations, time window) we considered a subarray, the second level trigger $(\boldsymbol{T} 2)$, is defined as $(2 \times 2) 4$-fold neighbouring stations $(/(3 \times 3)$ 9-fold neighbouring stations was also studied) and requiring to trigger at least a) 1 , b) 3 , and c) 5 hits on each station.

Fig.4(a) shows that the average time distribution of the 9 showers referred to first detected particle on each station of the subarray. The particles pass through the stations from bottom line to 

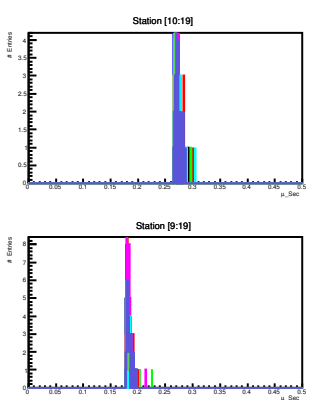

Station $[8: 19$

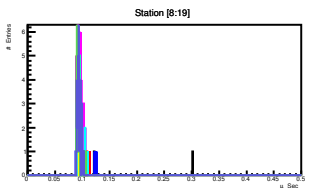

Station [::19]
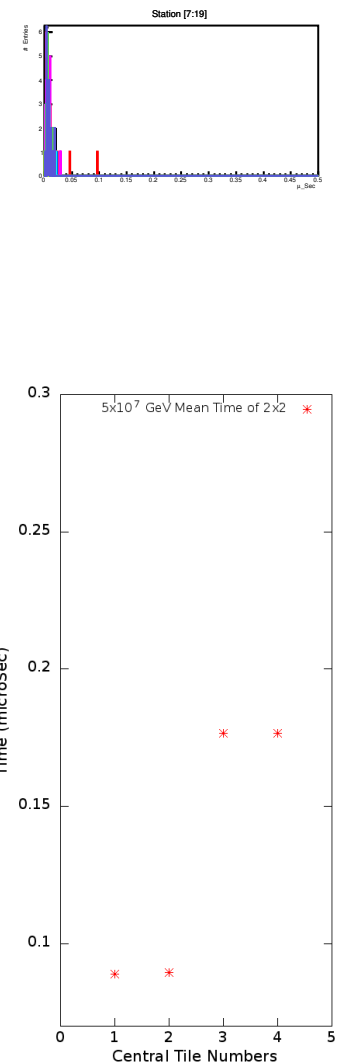
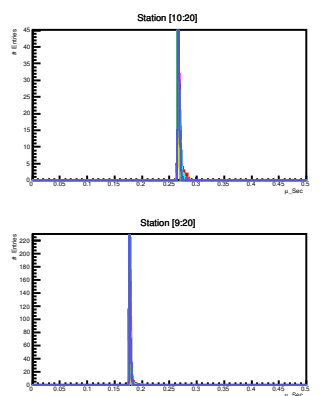

Station [18:20]

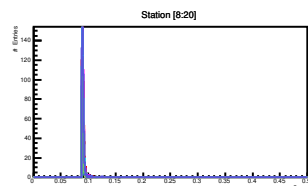

Station [7:20]
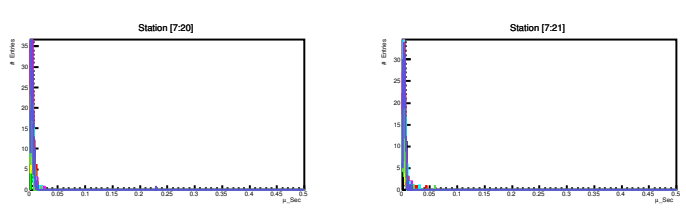

(a)

Delta Time Distribution on a Tower
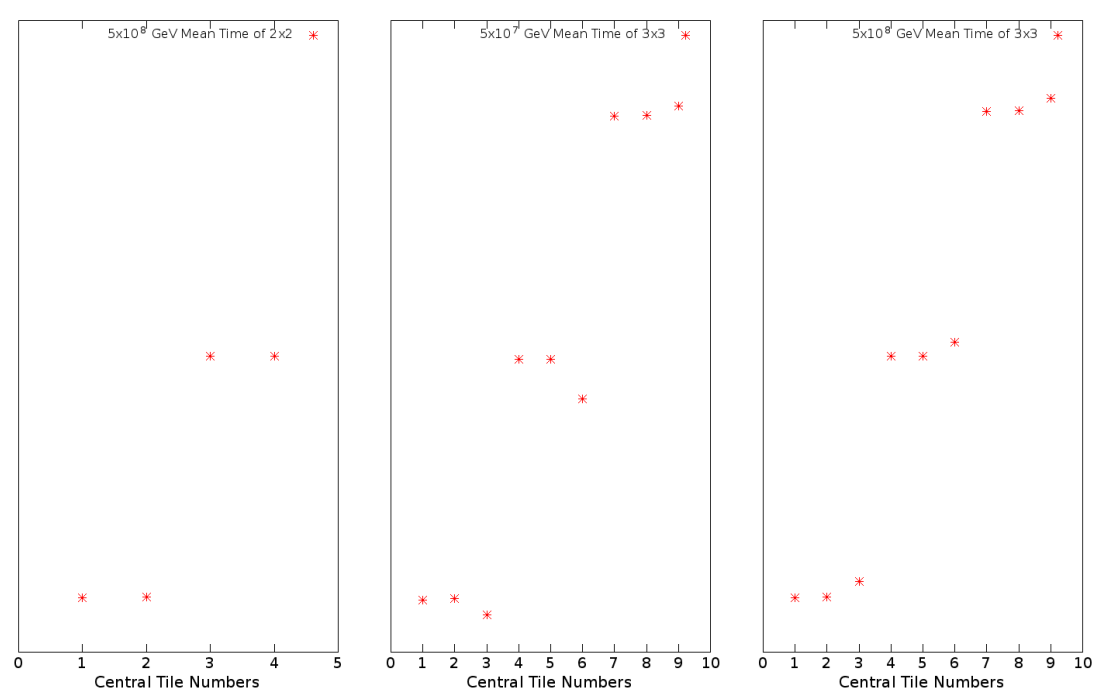
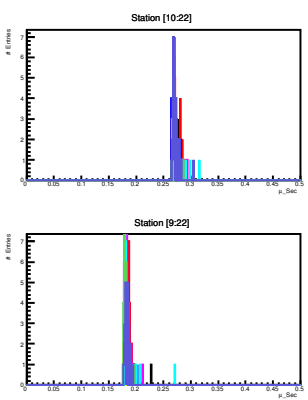

Station [1:22]

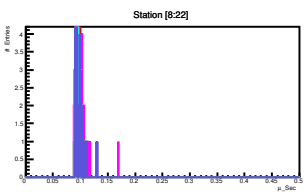

Station [7:22]

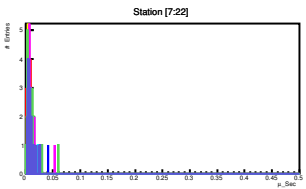

(b)

Figure 4: Timing efficiency of the subarrays.

the upper lines with $0.9 \mu s$ delay. The average time information of particles reading from subarray support the EAS front direction with this delay. Furthermore the shower front thickness in the center obtained as $0.45 \mathrm{~m}$ by the first and last hit particles to stations. In the Fig.4(b), the time distributions on the $2 \times 2$ and $3 \times 3$ stations belongs to $5 \times 10^{7} \mathrm{eV}$ and $5 \times 10^{8} \mathrm{eV}$ give an information about the average time of 9 different showers. For the $2 \times 2$ stations, timing efficiency changes with 
about $0.095 \mu \mathrm{s}$. In the $3 \times 3$ stations for lower energy, the particles hit the stations about $0.12 \mu \mathrm{s}$ and about $0.13 \mu$ s for the higher energy. The delay on the $3 \times 3$ cluster shows the EAS center shifted to the left side so this creates $5 n s$ delay on right detector. It means the curvature of the shower front greater on the right side. On the other hand the EAS center situated to the left side in higher energy.

Fig. 6 depicts that the triggering efficiencies for $\tau$-induced showers (9-showers per each setup) with fixed shower development length $(7 \mathrm{~km})$ and different $\tau$-lepton energies. The outcome of the Fig.6, the triggering efficiency of the array increases with the initial $\tau$-lepton energy. At 3 $\mathrm{km}$ shower development length, triggering probability is lower because of the produced showers may not fulfilled yet to reach its maximum development size. At the lower energy, the produced particles may not achieved to develop larger size showers and its secondaries were removed before detected by the detector stationing in the array. The trigger efficiency is risen since the primary lepton energy is higher that leads to generate more energetic secondary particles results in more stations to trigger with higher density of events.

In order to find the triggering probability as a function of required hits $(1,3$, or 5$)$ of stations ( $2 \times 2$ or $3 \times 3$ station), hundred showers were simulated and applied a fit at different conditions for $5 \times 10^{7} \mathrm{GeV} 5 \times 10^{8} \mathrm{GeV}$ energies, seen in Fig.5. As a result of this study, requiring at least 3 hits in each station of a subarray of $2 \times 2$ (cluster) with a total number of 6 clusters indicates $5 \times 10^{8} \mathrm{GeV}$ while 2 clusters of $2 \times 2$ sub array indicates $5 \times 10^{7} \mathrm{GeV}$.

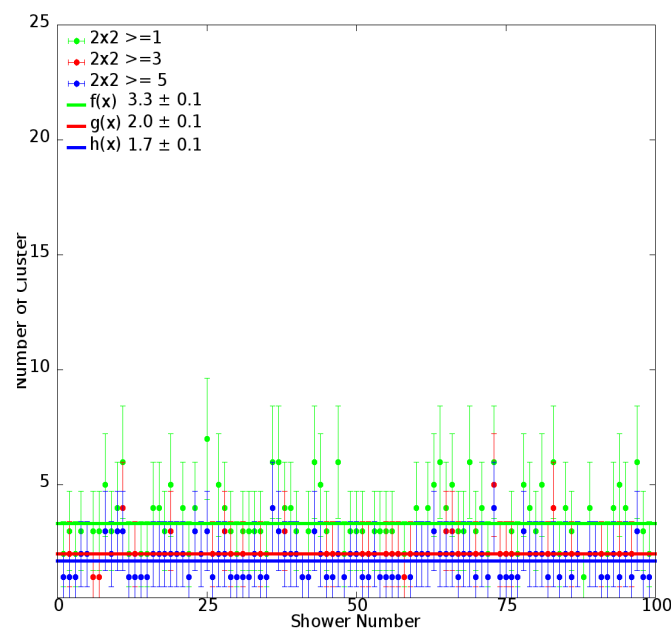

(a) $5 \times 10^{7} \mathrm{GeV}$

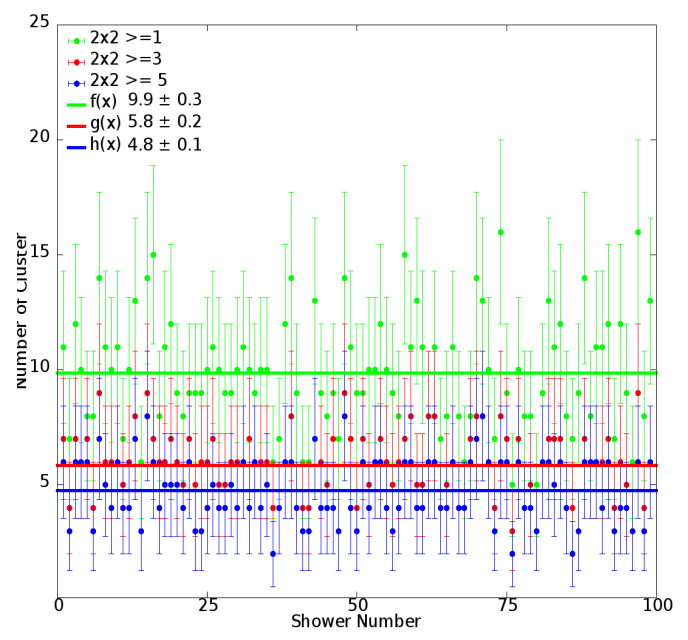

(b) $5 \times 10^{8} \mathrm{GeV}$

Figure 5: Average number of stations versus number showers for different required hit per station at fixed shower development length $(7 \mathrm{~km})$.

Fig.6 produced by the fit results of Fig.5 to clearly identify the behaviour of distributions. The Fig. 6 clearly depicts that the one digit increase in the energy is leading to increase about $70 \%$ in the number clusters. On the other hand, increasing in the required number of hits on each station cause to an exponential decrease on the the number clusters. 


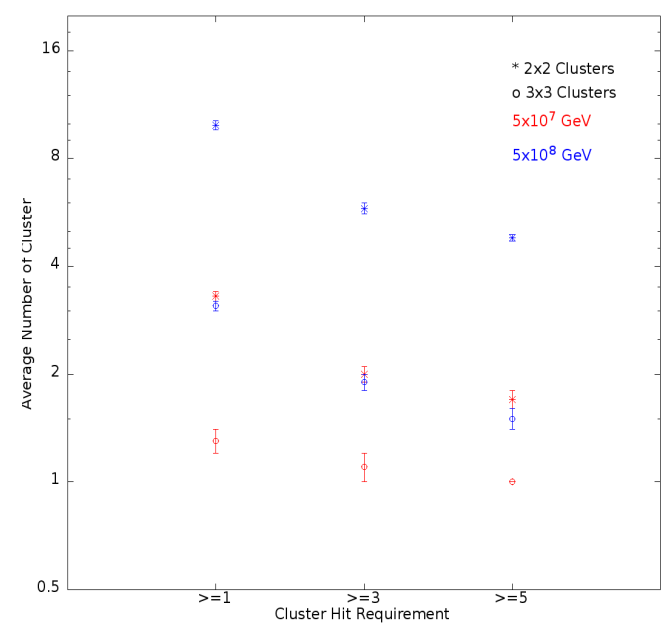

Figure 6: Average number of clusters triggered on the subarray $2 \times 2$ (star) and $3 \times 3$ (circle) stations. Red colour represents $5 \times 10^{7} \mathrm{GeV}$ energy, and blue is for $5 \times 10^{8} \mathrm{GeV}$ energy as a function of required hits at fixed $7 \mathrm{~km}$ shower development length of $\pi^{-} \pi^{0}$ decay mode.

\section{Summary and Outlook}

In this paper, MC studies of $\tau$-induced air showers based on CORSIKA simulation program on the proposed TAUWER array characteristics have been carried out. The trigger efficiency and identification in terms of the parameters: energy, shower development length have been estimated for the inclined array layout and different trigger conditions. The development of of shower, response of the array, trigger conditions, energy of the primary $\tau$-lepton particle are summed up within the triggering efficiency. The results of the studies point out the best shower development length to identify the primary $\tau$-lepton energy is between $5-7 \mathrm{~km}$ for the proposed array layout on the inclined plane for $\pi^{-} \pi^{0}$ decay mode. The TAUWER array proposed in this study is more efficient at higher energies than at lower energies. In the array setup, 3 different triggering conditions were studied on the neighbouring ( $2 \times 2$ or $3 \times 3$ - subarray) stations. As a result of this study, requiring at least 3 hits in each station of a subarray of $2 \times 2$ (cluster) with a total number of 6 clusters indicates $5 \times 10^{8} \mathrm{GeV}$ while 2 clusters of $2 \times 2$ sub array indicates $5 \times 10^{7} \mathrm{GeV}$. In order to find a candidate site for TAUWER array experiment, the study on a site that satisfy these requirements is in progress.

\section{Acknowledgement}

We acknowledge the financial support by the Scientific and Technological Research Council of Turkey (TUBITAK) (grant number: 114F138).

\section{References}

[1] E. Lorenz, Status of the 17-m MAGIC telescope, New Astron. Rev. 48 (2004) 339-344.

[2] T. C. Weekes, et al., VERITAS: The Very energetic radiation imaging telescope array system, Astropart. Phys. 17 (2002) 221-243. 
[3] J. A. Hinton, The Status of the H.E.S.S. project, New Astron. Rev. 48 (2004) 331-337.

[4] W. D. Apel, et al., The KASCADE-Grande experiment, Nucl. Instrum. Meth. A620 (2010) 202-216.

[5] F. Halzen, S. R. Klein, IceCube: An Instrument for Neutrino Astronomy, Rev. Sci. Instrum. 81 (2010) 081101.

[6] M. Actis, et al., Design concepts for the Cherenkov Telescope Array CTA: An advanced facility for ground-based high-energy gamma-ray astronomy, Exper. Astron. 32 (2011) 193-316.

[7] T. Huege, et al., The LOPES experiment - recent results, status and perspectives, Nucl. Instrum. Meth. A662 (2012) S72-S79.

[8] M. P. van Haarlem, et al., LOFAR: The LOw-Frequency ARray, Astron. Astrophys. 556 (2013) A2.

[9] M. G. Aartsen, et al., Evidence for High-Energy Extraterrestrial Neutrinos at the IceCube Detector, Science 342 (2013) 1242856.

[10] D. Fargion, A. Aiello, R. Conversano, Horizontal tau air showers from mountains in deep valley. traces of uhecr neutrino tau

[11] E. Zas, Neutrino detection with inclined air showers, New Journal of Physics 7 (2005) 130-130.

[12] M. Iori, H. Denizli, A. Yilmaz, F. Ferrarotto, J. Russ, Electron-muon identification by atmospheric shower and electron beam in a new eas detector concept, The Astrophysical Journal 801 (2) (2015) 140.

[13] M. Iori, I. O. Atakisi, G. Chiodi, H. Denizli, F. Ferrarotto, M. Kaya, A. Yilmaz, L. Recchia, J. Russ, Sipm application for a detector for uhe neutrinos tested at sphinx station, Nucl. Instrum. Methods Phys. Res. Sect. A-Accel. Spectrom. Dect. Assoc. Equip. 742 (2014) 265-268.

[14] S. Ritt, R. Dinapoli, U. Hartmann, Application of the drs chip for fast waveform digitizing, Nuclear Instruments and Methods in Physics Research Section A: Accelerators, Spectrometers, Detectors and Associated Equipment 623 (1) (2010) 486-488.

[15] Z. Was, S. Jadach, TAUOLA Monte Carlo for tau decays, in: Proceedings, 26th International Conference on High-energy Physics (ICHEP 92): Dallas, Texas, USA, August 6-12, 1992, 1992, pp. 1777-1780.

[16] D. Fargion, Arrays is space to detect upward tau and highest altitude showers

[17] D. Heck, G. Schatz, T. Thouw, J. Knapp, J. N. Capdevielle, CORSIKA: A Monte Carlo code to simulate extensive air showers.

[18] S. Ostapchenko, Nonlinear screening effects in high energy hadronic interactions, Phys. Rev. D74 (1) (2006) 014026. 